\title{
Impacto económico de los Pueblos Mágicos desde la perspectiva de los ciudadanos: Izamal, Yucatán
}

\section{Economic impact of the Magical Towns from the perspective of citizens: Izamal, Yucatán}

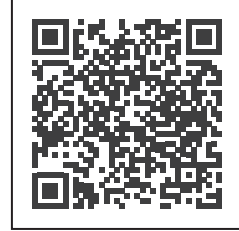

Palabras clave:

ciudadanía, dinámica económica, economía, población, servicios, turismo.

Keywords: Citizenship; Economic Dynamics; Economy; Population; Services; Tourism.

Códigos JEL: Z320 Turismo y desarrollo; D62 Economía del bienestar

Artículo de investigación

Fecha de recepción:

14 de marzo de 2021

Fecha de aprobación:

24 de agosto de 2021

Fecha de publicación:

16 de septiembre de 2021

Creative Commons

Reconocimiento-NoComercial-SinObraDerivada 4.0 Internacional

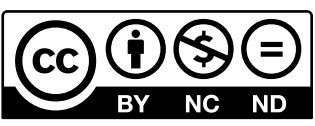

Karina Concepción González-Herrera';

Aurea Licet Castillo-Gallegos²; Karla Gabriela Gómez-Galaz; José Jesús Hernán Negrón-Noh

\section{Resumen}

El municipio de Izamal ha presentado un dinamismo importante desde el nombramiento efectuado en 2002 como Pueblo Mágico por la Secretaría de Turismo. Esta circunstancia condujo a una transición económica del municipio, lo cual ha impactado en la economía y en las actividades que realiza la ciudadanía, siendo estas escasamente abordadas para la implementación de acciones que incidan en el crecimiento económico del municipio.

El objetivo es identificar el impacto económico que representa el distintivo de Pueblo Mágico desde la perspectiva de la ciudadanía.

El método integrado a este artículo es de corte cuantitativo descriptivo, considerando el 95\% de confianza para la determinación del tamaño muestral, con respecto a los instrumentos recabados;

1 Licenciada en Administración. Maestra en Ciencias en Planificación de Empresas y Desarrollo Regional. Doctora en Desarrollo Regional, Universidad Tecnológica Metropolitana, México. karina.gonzalez@utmetropolitana.edu. mx, ORCID: https://orcid.org/0000-0002-1743-2614

2 Licenciada en Economía. Maestra en Agronegocios, Universidad Tecnológica Metropolitana, México. aurea.castillo@utmetropolitana.edu.mx, ORCID: https://orcid.org/0000-0003-4545-4957

3 Contador público. Maestra en Finanzas, Universidad Tecnológica Metropolitana, México. karla.gomez@utmetropolitana.edu.mx, ORCID: https:// orcid.org/0000-0002-0640-0756

4 Licenciado en Economía. Maestro en Administración de Recursos Humanos. Doctor en Administración, Universidad Tecnológica Metropolitana, México. jose.negron@utmetropolitana.edu.mx, ORCID: https://orcid.org/00000002-9216-6061 


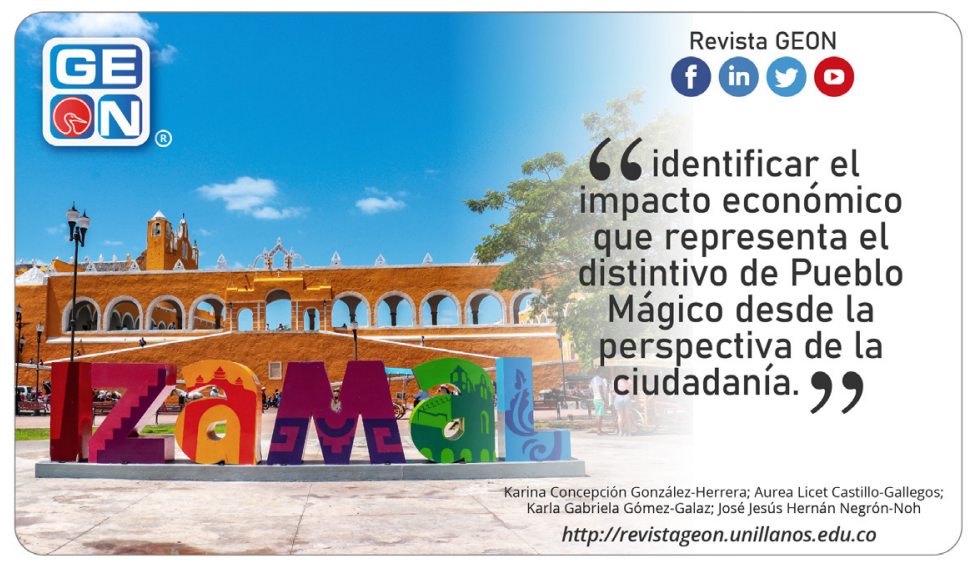

un 0,051 relativo al error de estimación, con una proporción a favor (p) y en contraparte (q) del 0,5, respectivamente.

La población ha transitado primeramente de las actividades económicas tradicionales a la oferta de servicios, cuya dirección es la actividad turística. Del mismo modo, se observó una mejoría en la infraestructura física de los edificios de la zona centro de la localidad, la economía inmersa desempeñó un papel fundamental con la participación de los empresarios, comerciantes y principalmente de la población (ciudadanía) en conjunto. La afluencia turística representa beneficios económicos susceptibles para la economía de la población.

Se identificó que el comercio de artesanías en 2021 no destaca un incremento significativo con relación al 2019, contrariamente al comercio de ropa típica y de los servicios de alimentos, como productos dirigidos hacia el turismo, que permiten la mejoría de ingresos de los ciudadanos.

Para concluir, se puede mencionar que la difusión es un elemento fundamental para dar a conocer las acciones dirigidas hacia los ciudadanos del Programa Pueblos Mágicos que inciden en resultados tangibles para ellos.

Contribuye a la generación de propuestas encaminadas a mejorar la percepción del programa y la implicación de la ciudadanía en él. 
Palabras clave: ciudadanía, dinámica económica, economía, población, servicios, turismo.

Códigos JEL: Z320 Turismo y desarrollo; D62 Economía del bienestar

\section{Abstract}

Since 2002, following its designation as a Magical Town by the Ministry of Tourism, the municipality of Izamal has experienced a significant dynamism. This led to an economic transition of the municipality, which has had an impact on the economy and on the activities carried out by the citizens, which have been scarcely addressed for the implementation of actions that have an impact on the economic growth of the region.

The purpose of this study is to identify the economic impact of the Magical Town distinction from the citizens' perspective.

A descriptive quantitative method was used for this article, taking into account a 95\% confidence level for the calculation of the sample size, regarding the instruments collected; an estimation error of 0.051, with a sample proportion in favor ( $p$ ) and against ( $q$ ) of 0.5 , respectively.

First of all, the population has shifted from traditional economic activities to providing services, heading towards tourism. In the same way, an improvement in the buildings' physical infrastructure was observed in the downtown area of the locality, the immersed economy played a fundamental role with the participation of the businessmen, merchants and mainly of the population (citizens) as a whole; therefore, the

Cómo citar este artículo / Toreference this article:

González Herrera, K. C., Castillo Gallegos, A. L., Gómez-Galaz, K. G., \& Negrón-Noh, J. J. H. (2021). Impacto económico de los Pueblos Mágicos desde la perspectiva de los ciudadanos: Izamal, Yucatán. Revista GEON (Gestión, Organizaciones Y Negocios), 8(2), e-306. https://doi. org/10.22579/23463910.306 tourist affluence represents susceptible economic benefits for the population's economy.

In 2021, it was identified that the handicraft commerce does not have a significant increase in relation to 2019, contrary to the commerce of typical clothing and food services, as products directed towards tourism, which allows the improvement of citizens' income. 
Cómo citar este artículo / Toreference this article:

González Herrera, K. C., Castillo Gallegos, A. L., Gómez-Galaz, K. G., \& Negrón-Noh, J. J. H. (2021). Impacto económico de los Pueblos Mágicos desde la perspectiva de los ciudadanos: Izamal, Yucatán. Revista GEON (Gestión, Organizaciones Y Negocios), 8(2), e-306. https://doi. org/10.22579/23463910.306
To conclude, it can be mentioned that dissemination is a fundamental element to promote the actions directed towards the Magical Towns Program's citizens, which leads to tangible results for them.

It contributes to the formulation of proposals directed to improve the perception of the program and the involvement of citizens in it.

Keywords: Citizenship; Economic Dynamics; Economy; Population; Services; Tourism.

JEL codes: Z320 Tourism and development; D62 Welfare economics

\section{Introducción}

El presente estudio se vincula con la actividad económica del turismo, la cual se fortalece una vez el municipio de Izamal, Yucatán es nombrado Pueblo Mágico. Allí se destacan el comercio y los servicios; sin embargo, se desconocen las actividades económicas que quedaron relegadas a partir de este nombramiento del territorio yucateco. También existe poca noción de las actividades económicas alternas que los pobladores desempeñan actualmente en la localidad. Para identificar la percepción de la ciudadanía con relación a esta dinámica económica, resulta relevante su opinión para establecer propuestas encaminadas a la mejora, con el fin de que el beneficio que perciba la población se incremente de manera gradual. El Programa Pueblos Mágicos es un fuerte impulsor del turismo, que influye en la activación de las economías locales, promoviendo la generación de empleos y, a su vez, estimulando al emprendedor. Para ello, se requieren conocer las necesidades inherentes a la economía de la población, puesto que cada localidad necesita diversos factores para que sea percibida una mejoría local, de acuerdo con Güidi (2016). Debido a que no se Iogran observar aquellos beneficios económicos de la población, el objeto de este estudio es identificar el impacto de los Pueblos Mágicos desde la perspectiva de la ciudadanía, lo que permitirá plantear propuestas de acción encaminadas a la dinámica económica poblacional que lleve a incidir en las decisiones.

\section{Contexto teórico}

El turismo es una de las actividades económicas más prevalentes en México, motivo por el cual existen diferentes programas en los tres distintos niveles de gobierno que impulsan el crecimiento y el desarrollo de este (Pérez \& Antolín, 2016).

El turismo es un fenómeno mundial en crecimiento a partir del cual se 
implementan planes y programas de promoción que prometen ser motor de desarrollo económico. Instituciones internacionales, como el Consejo Mundial de Viajes y Turismo (CMVT) y la Organización Mundial del Turismo (OMT), afirman que este proceso social favorece el crecimiento económico global a través de la creación de nuevos empleos, exportaciones y la estimulación de la diversificación productiva (Flores-Pacheco, 2019, p. 5).

Es por ello por lo que, en busca de un mejor aprovechamiento de los recursos naturales, las tradiciones, la cultura y los atractivos turísticos, entre otros, se crea el Programa de Pueblos Mágicos en México a través del desarrollo comunitario para el beneficio de los pobladores.

En la Guía de Incorporación y Permanencia Pueblos Mágicos, la Secretaría de Turismo (SECTUR, 2014, p. 3) menciona que Pueblos Mágicos es un programa de política turística federal que se dirige a las localidades como una marca distintiva del turismo de México. Se busca que este programa se mantenga a través del cumplimiento de las reglas de operación, lo cual estimula el turismo en las localidades y contribuye a mejorar el empleo, entre otros.

El objeto del programa es fomentar el desarrollo sustentable de las localidades poseedoras de atributos de singularidad, carácter y autenticidad a través de la puesta en valor de sus atractivos, representados por una marca de exclusividad y prestigio, teniendo como referencia las motivaciones y necesidades del viajero actual (SECTUR, 2014, p. 4).
Un Pueblo Mágico es una localidad que conserva su herencia histórica, cultural y natural, lo que se convierte en áreas de oportunidad y en un atractivo para el turismo, siendo estos escenarios solicitados y/o deseados por el turista SECTUR (2017, p.4, citado en Alcocer-Kuk et al., 2019, p. 9).

El Programa Pueblos Mágicos se crea en 2000 en México e inicia en 2001 con dos municipios, Huasca de Ocampo (Hidalgo) y Real del Catorce (San Luís Potosí). En 2012 suman 83 (Fernández, 2016), para 2019 son 126 y en 2020 llegan a 132.

En un estudio de competitividad, realizado por la Secretaría de Turismo Federal (SECTUR), la Secretaría de Fomento Turístico del Gobierno del Estado de Yucatán (SEFOTUR) y la Universidad Autónoma de Yucatán (UADY) (s. f.), como parte de los criterios para la certificación del nombramiento como Pueblo Mágico, se indica que "la localidad deberá acreditar que ha recibido apoyos directos de programas institucionales, estatales y/o federales que contribuyen y benefician directa o indirectamente su actividad turística, detallando la inversión y el impacto de los beneficios" (p. 13).

El programa incide en la mejora de los municipios en cuanto a pintura e infraestructura, con recursos federales destinados para ello. Estas inversiones en infraestructura, mantenimiento y capacitación benefician de manera directa a los pobladores del municipio, incluidas las familias, los comercios y la población en general, a través de su participación en las 
diferentes actividades planificadas y desarrolladas por las instituciones de turismo local, estatal y federal. Estas acciones generan una derrama económica para la comunidad, tal como lo menciona Flores-Pacheco (2019, p. 9) en los resultados que obtuvo en un estudio realizado en un Pueblo Mágico ubicado en Oaxaca, en el que se llegó a la conclusión de que "la principal motivación para participar en las actividades del proceso turístico es mejorar el desarrollo económico de la comunidad", de acuerdo con la información proporcionada por los sujetos de estudio de San Pablo Villa de Mitla. Es entonces cuando la actividad turística contribuye para fomentar y lograr un crecimiento económico local.

De acuerdo con Cabrera (2017), "el desarrollo local recoge el sentido social y productivo orientado a mejorar la calidad de vida de una población en un territorio (local) con características culturales, productivas y sociales particulares frente a un escenario más amplio" (p. 119). El desarrollo local constituye un proceso estructurado de perspectivas económicas, políticas, socioculturales y físicas a través del cual se puede alcanzar el bienestar sumado de la población. Constituye un concepto interrelacionado, donde los propios actores sociales participan directamente en la construcción de alternativas de solución a problemáticas comunes, así como en la definición de sus propios procesos de desarrollo basados en las necesidades, expectativas e intereses compartidos. Por lo tanto, para que pueda darse un desarrollo local, es indispensable la participación de cada uno de los actores involucrados: los pobladores, las empresas y las autoridades (Pérez \& Antolín, 2016). De acuerdo con estos mismos autores, en un estudio realizado en un Pueblo Mágico en Querétaro, los pobladores enfatizaron en el aspecto de que ninguno de ellos como residentes participó en algún tipo de consulta para incorporar la localidad al Programa de Pueblos Mágicos. No obstante, consideran que es bueno para el lugar, principalmente en el ámbito económico, ya que ha traído derrama al pueblo. En cambio, por el lado social y medioambiental opinan que no se ha tenido un impacto benéfico, sino al contrario. La demanda turística ha hecho que se creen suficientes fuentes de empleo en el destino, lo que también ha contribuido a que pobladores de localidades vecinas (en las cuales no hay suficientes oportunidades de trabajo) emigren a la ciudad. Mencionan que los que más se benefician son las empresas y el gobierno y, en conclusión, como menciona Segovia et al. (2019, p. 303), "la denominación de Pueblo Mágico sí ha contribuido a generar desarroIlo en la localidad de Bernal, mas, no obstante, aún existe una situación de rezago y desigualdad dentro de la población". Con base en los resultados de su investigación, Pérez y Antolín (2016, p. 238) identificaron que para el desarrollo local la situación es difícil, puesto que hay una ausencia de coordinación y cooperación interna entre la población y el H. Ayuntamiento. Aunque los recursos e iniciativas son valiosas, los beneficios son para un sector reducido de la población, lo cual obstruye el desarrollo de la localidad en su conjunto. 
Como puede observarse, en muchos de los casos, los ciudadanos de la población no tienen acceso a la información y a los beneficios que pueden obtener de este programa, hasta el punto de considerarse excluidos. Es indispensable un análisis y estudio que permita determinar áreas de oportunidad y mejora que contribuyan al cumplimiento de los objetivos del programa. Abordando los datos que se refieren al municipio de Izamal, objeto de la investigación realizada, se observa que fue nombrado Pueblo Mágico en 2002. Se localiza en Yucatán, en las coordenadas 20.93138989.017778, colinda con diversos municipios: al norte con Tekal de Venegas, Tepakán y Tekantó; al sur con Xocchel, Kantunil y Sudzal; al este con Tunkás; por último, al oeste con Honctún (SECTUR, 2014, Población, párrafo 1). Para 2020, de acuerdo con el Censo de Población y Vivienda del Instituto Nacional de Estadística y Geografía (INEGI), la población total equivale a 28.555 habitantes, identificándose un incremento del 9,9\% con respecto al decenio anterior. Izamal se caracteriza por sus atractivos culturales, su gastronomía, usos y costumbres, tradiciones, sitios arqueológicos, entre otros. De acuerdo con el Programa de Destinos México, en su Guía Turística de Yucatán (2014), Izamal es conocida como la ciudad de las colinas, ya que vestigios arqueológicos permanecen en el centro de la ciudad. También se le llama la ciudad de las tres culturas (SECTUR, 2014, p. 15). El distintivo de la localidad son todas las casas, comercios e iglesias que se encuentran pintados de color amarillo.
Se considera un importante centro religioso debido a que se encuentra el exconvento franciscano San Antonio de Padua, templo de la Virgen de la Inmaculada Concepción. El atrio del convento es considerado uno de los más grandes en América Latina.

\section{Materiales y métodos}

El enfoque de investigación efectuado es cuantitativo (Hernández et al., 2014), ya que en su diseño se selecciona como instrumento de investigación el cuestionario, documento que permite el análisis e interpretación de los datos recabados, cuyas ventajas en la utilización son (Barbero et al., 2006; Tourón, 1989; Verdugo \& Cal, 2010 citado en Lacave et al., 2016): poseen propiedades psicométricas, que estudian los pasos estadísticos, los cuales permiten comprobar su validez y fiabilidad de acuerdo con las variables establecidas; pueden ser originales o adaptados y existe material suficiente en línea e impreso para perfeccionar los ítems integrados en estos. Para ello, se diseñó el instrumento dirigido a los ciudadanos con 21 ítems, considerando de esta forma abarcar la percepción de los actores involucrados en la dinámica que presentan los Pueblos Mágicos en México. En particular, los sujetos de estudio requerían contar con la mayoría de edad para que sus respuestas se consideraran válidas. El ámbito de actuación fue el municipio de Izamal.

Se determinó el tamaño muestral considerando la siguiente fórmula para poblaciones finitas: 


$$
\begin{aligned}
& n=\frac{\left(N Z^{2} p q\right)}{\left(d^{2}(N-1)+Z^{2} p q\right)}=364 \text { cuestionarios } \\
& N=26801(\text { INEGI, 2020) } \\
& Z^{2}=1.96=3.8416 \\
& d^{2}=0.051=0.002601 \\
& p=0.5 \\
& q=0.5
\end{aligned}
$$

En la aplicación directa se recabaron 369 cuestionarios que se obtuvieron mediante entrevistas dirigidas. El Alpha de Cronbach fue del 0,90 con una alta consistencia de confiabilidad de los ítems. Para la validez del cuestionario, se procedió a la valoración por parte de los expertos en la temática. Con las observaciones respectivas se logró precisarlo para su aplicación.

\section{Resultados}

Las características de los sujetos de estudio que dieron respuesta a los instrumentos de investigación, mediante la técnica de encuesta dirigida, son las siguientes: la edad oscila entre 18 y 74 años o más, se obtuvo mayor representatividad en los ciudadanos de 30 a 34 años, con una media aritmética de 4,69. Por otra parte, el género de mayor representatividad fue el masculino, con una media aritmética de 1,42. Tanto la edad como el sexo fueron negativos con -0,389, lo que indica que es asimétrica a la izquierda, con una escolaridad promedio de secundaria de 2,31. Así mismo, posee una curtosis denominada platicúrtica, cuyos resultados fueron negativos con -1,065. Para el caso de los datos de la asimetría, el resultado equivale a 0 , lo que indica que la distribución es simétrica (tabla 1).

Con base en el comportamiento de los datos asociados a la edad y al nivel educativo, se puede identificar que la secundaria concluida es la que tiene mayor representatividad entre los ciudadanos de 35 a 39 años (figura 1). Un menor rango de edad y el nivel educativo se asocia con menor posibilidad de ingresos en los ciudadanos estudiados, al desempeñar las actividades relacionadas con el turismo en el municipio de Izamal.

Al ser un municipio turístico y con base en las características territoriales de este, al momento de cuestionar a los ciudadanos sobre las actividades alternas al turismo (como la pesca) a manera de una opción de ingresos en las diferentes temporadas del turismo, especialmente en la temporada de baja afluencia turística, se obtuvo

\begin{tabular}{|c|c|c|c|c|c|c|c|}
\hline \multirow{2}{*}{ Datos } & \multirow{2}{*}{\begin{tabular}{|c|}
$\mathbf{N}$ \\
Estadístico
\end{tabular}} & \multirow{2}{*}{$\begin{array}{c}\text { Media } \\
\text { Estadístico }\end{array}$} & \multirow{2}{*}{\begin{tabular}{|c|}
$\begin{array}{c}\text { Desviación } \\
\text { estándar }\end{array}$ \\
Estadístico
\end{tabular}} & \multicolumn{2}{|c|}{ Asimetría } & \multicolumn{2}{|c|}{ Curtosis } \\
\hline & & & & Estadístico & $\begin{array}{c}\text { Error } \\
\text { estándar }\end{array}$ & Estadístico & $\begin{array}{c}\text { Error } \\
\text { estándar }\end{array}$ \\
\hline Edad & 369 & 4,69 & 3,427 & 0,640 & 0,127 & $-0,517$ & 0,253 \\
\hline Sexo & 369 & 1,42 & 0,580 & $-0,389$ & 0,127 & $-0,733$ & 0,253 \\
\hline Escolaridad & 369 & 2,31 & 1,604 & 0,000 & 0,127 & $-1,065$ & 0,253 \\
\hline N válido (por lista) & 369 & & & & & & \\
\hline
\end{tabular}
que un 70,7\% presenta un bajo interés o las descarta totalmente. Así mismo, se presentó un bajo interés

Tabla 1.Análisis descriptivo de los datos generales de los sujetos de estudio

Fuente: elaboración propia de los autores. 
en realizar actividades relativas a la agricultura/ganadería, con el 41,2\%, debido a que desempeñando estas actividades se obtienen ingresos bajos y, además, hay poca existencia de terrenos para esta actividad. En el caso específico de la agricultura, cabe destacar que el tipo de suelo, que es mayoritariamente cárstico, impide mejores resultados en la cosecha. La actividad industrial también presenta un bajo interés, con el 35,2\%, ya que, en la población, el proceso industrial no se ha impulsado totalmente, toda vez que sería distante a la localidad el insertarse laboralmente. Se registra un predominante $58,3 \%$ de interés de los sujetos de estudio en realizar actividades relacionadas con el comercio y los servicios (figura 2). Lo anterior permitió identificar el tránsito de las actividades tradicionales del municipio, como la agricultura y la ganadería, hacia el comercio y los servicios asociados al turismo, que reflejan un mayor ingreso para los ciudadanos.
Al indagar sobre otras actividades económicas desarrolladas, asociadas al turismo en la localidad, el 76,7\% de los ciudadanos indicaron que no se han desarrollado otras actividades económicas relacionadas con el turismo. De acuerdo con el género, las respuestas reflejan que el 35,5\% de las mujeres y el 37,9\% de los hombres no realizan actividades alternas al turismo. El 10,6\% de los hombres y el 9,8\% de las mujeres indicaron que sí se han presentado esos cambios (siendo esta una baja percepción con respecto a la totalidad cuestionada). Las actividades económicas tradicionales como el bordado y el urdido, entre otras, que desempeñaban los ciudadanos de Izamal, son también las actividades que transitaron al turismo, es decir, se dejaron de realizar y se optó por el comercio de alimentos o servicios turísticos, puesto que esta es una forma de obtener mayores ingresos (figura 3). También se logró identificar el abandono del campo (agricultura y ganadería).

Figura 1. Rango de edad según la escolaridad de los sujetos de estudio de Izamal.

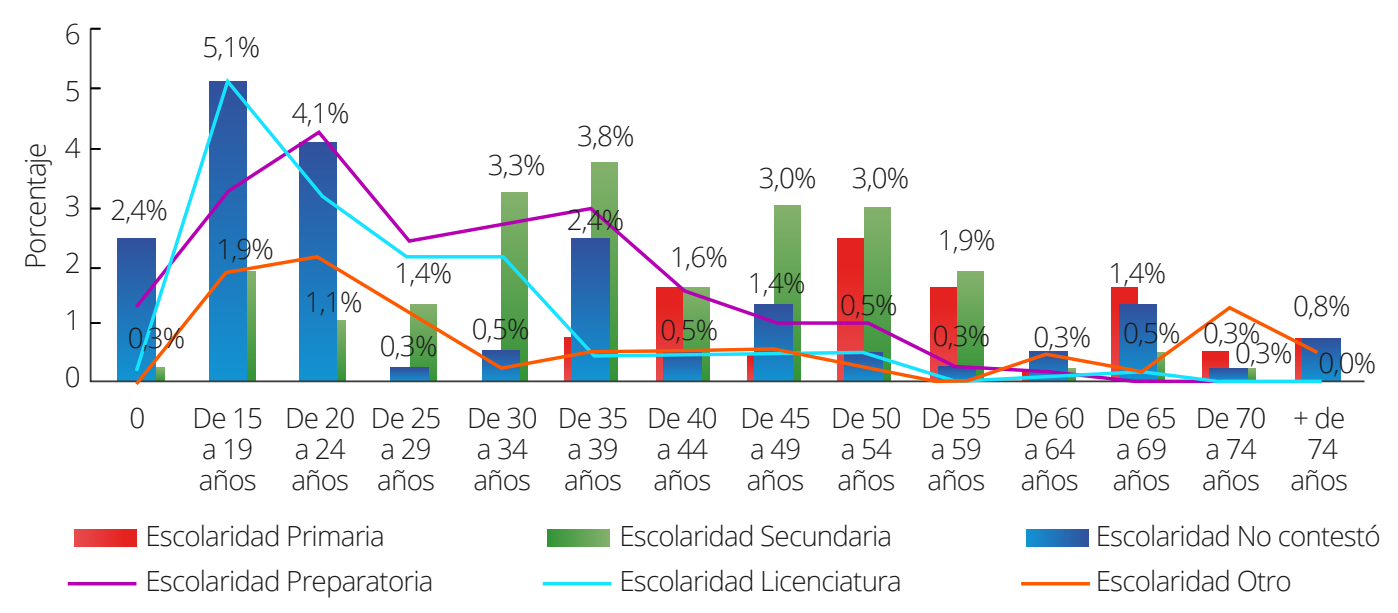

Fuente: elaboración propia de los autores. 
Figura 2. Interés de la comunidad en realizar actividades asociadas al turismo desde la perspectiva del comercio/servicios, industria, pesca y agricultura/ganadería.

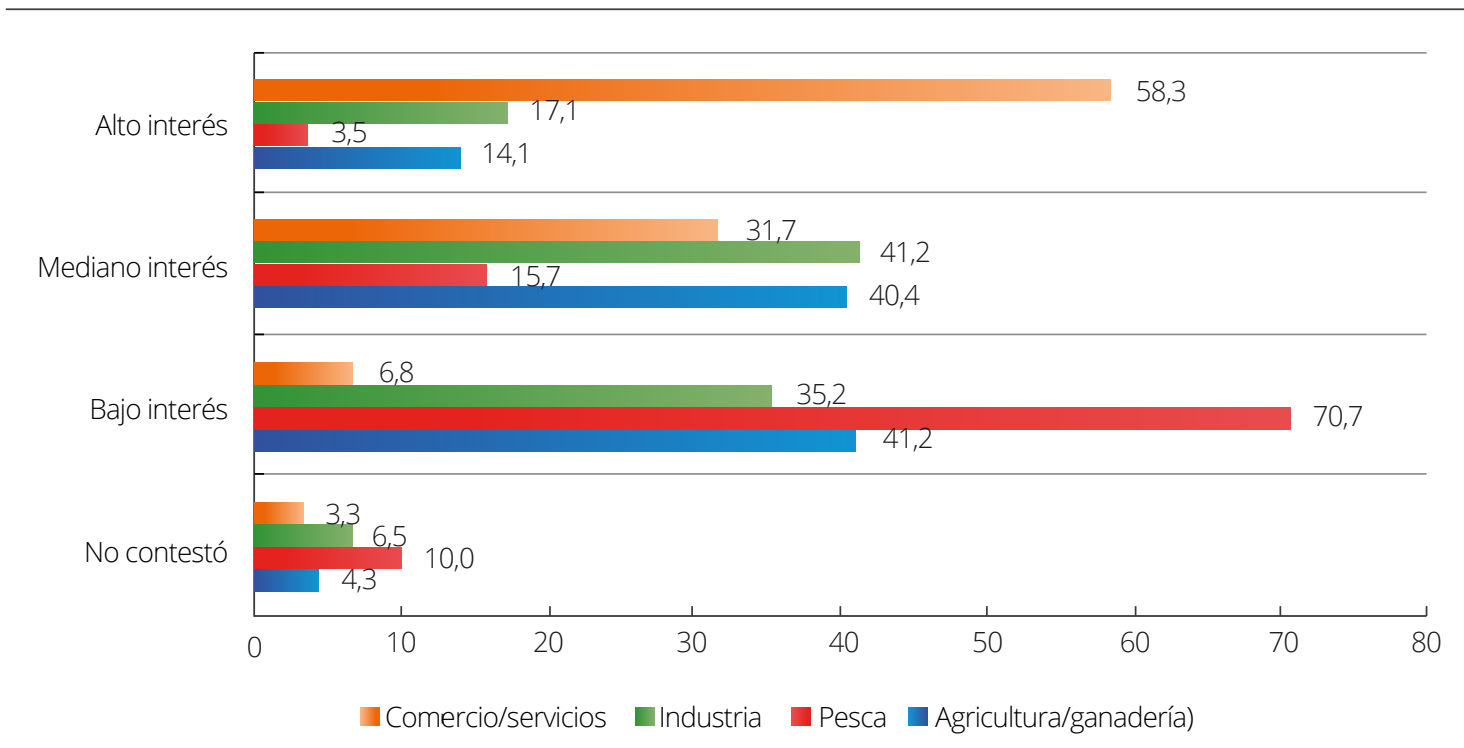

Fuente: elaboración propia de los autores.

Figura 3. Perspectiva del cambio de actividad económica según el género.

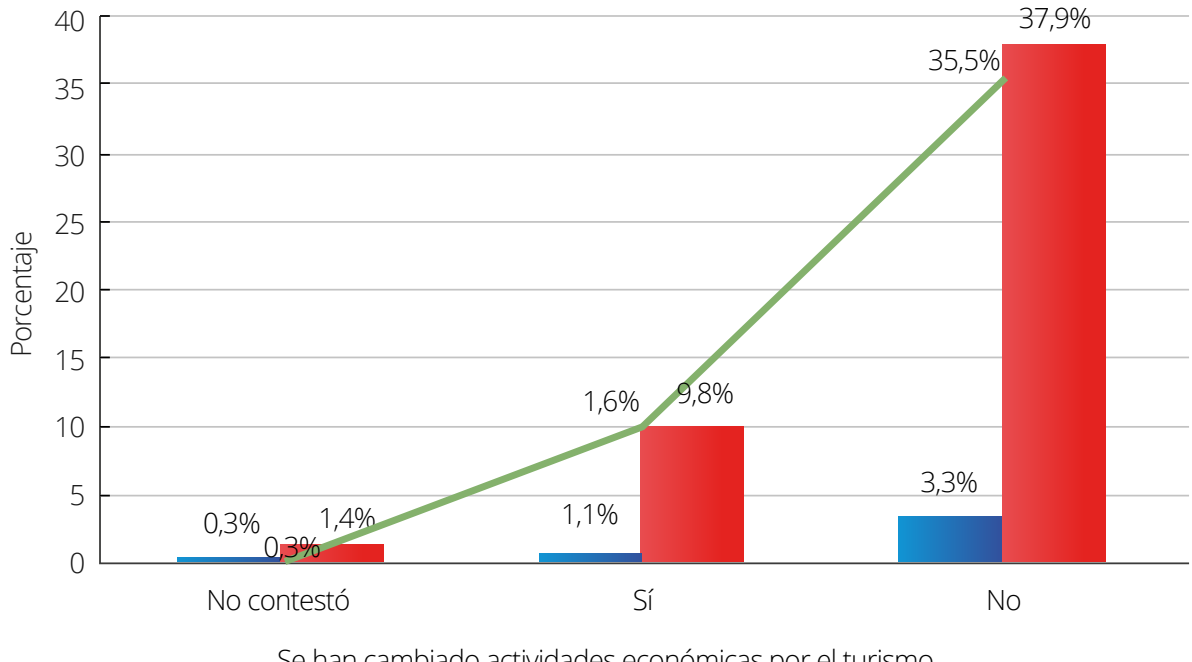

Se han cambiado actividades económicas por el turismo

Masculino Femenino No contestó

Fuente: elaboración propia de los autores. 
Considerando los beneficios que produce el turismo, desde un enfoque de género, el 32\% de las mujeres y el $31,7 \%$ de los hombres mencionaron que este se dirige primeramente hacia los empresarios y comerciantes. El $18,4 \%$ hizo énfasis en que se beneficia toda la comunidad (el 8,1\% de las mujeres y el 9,2\% de los hombres) y el 13,2\% indicó que el beneficiario directo es la autoridad o autoridades municipales (el 6,2\% de las mujeres y el $7 \%$ de los hombres). El resto de la población encuestada no dio respuesta (figura 4). Cabe destacar que, a través de las actividades tradicionales, los ingresos de los ciudadanos no lograban alcanzar un salario mínimo (SM), lo cual presenta una transición positiva económica a través del nom- bramiento del municipio como Pueblo Mágico; sin embargo, esto no se produce para toda la población.

Seguidamente, el $46,9 \%{ }^{5}$ de los ciudadanos indican que la situación económica del municipio es percibida como buena y muy buena. Sin embargo, el 6,8\% no ha observado cambios en los ingresos y la economía de los pobladores (figura 5). Desde esta perspectiva, el destinatario principal de los ingresos económicos es el empresario y los comerciantes, pero con respecto a la infraestructura del municipio, el cuidado de la imagen y limpieza de este ha mejorado, principalmente en el primer cuadro de la ciudad de Izamal, a partir de su nombramiento como Pueblo Mágico.

5 Porcentaje equivalente a la suma de los datos acumulados del género femenino y masculino.

Figura 4. Dirección de los beneficios que produce el turismo del Pueblo Mágico de Izamal.

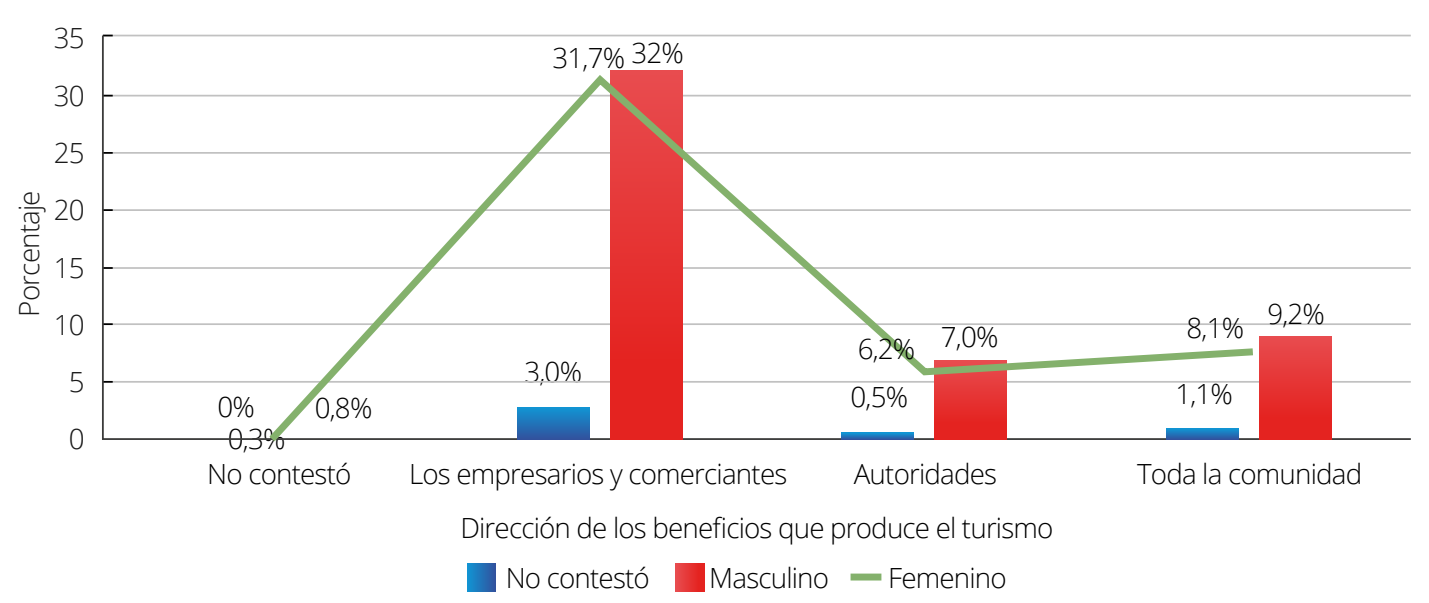

Fuente: elaboración propia de los autores. 
Figura 5. Valoración de la situación económica del municipio a partir del nombramiento como Pueblo Mágico.

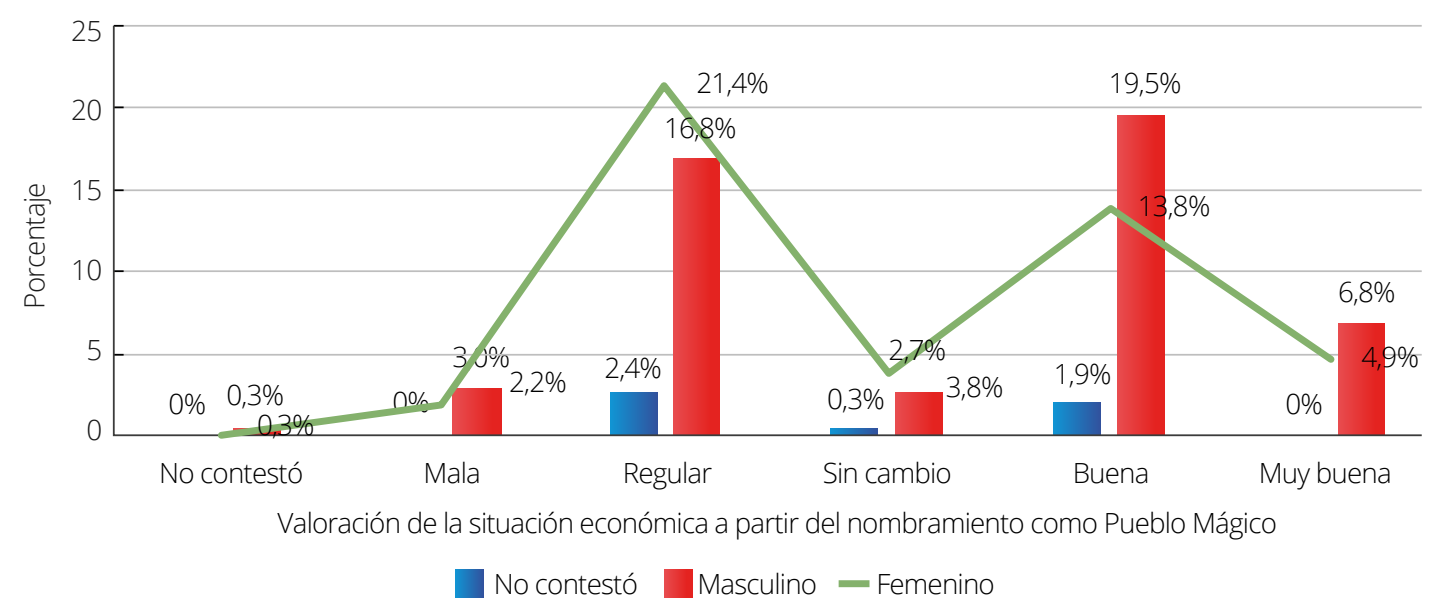

Fuente: elaboración propia de los autores.

Así mismo, se obtuvo que el 89,4\% mencionó que a partir del nombramiento se ha incrementado el flujo de turistas y visitantes a la localidad.

\section{Discusión}

De acuerdo con el. Consejo Nacional de Población (CONAPO, 2015), Yucatán cuenta con un índice de marginación (IM) de 0,51, catalogado con un grado de alto. Izamal presenta un IM de -0,116 (grado medio) (CONAPO, 2015), en donde la actividad preponderante es el turismo, el cual ha estimulado la economía de los habitantes a través del turismo cultural, el turismo religioso y la gastronomía típica de la localidad.

El Programa Pueblos Mágicos sigue siendo una apuesta para detonar las economías locales, destacando la singularidad de cada pueblo, lo cual estimula los productos turísticos e incrementa el flujo de los turistas (Ramos, 2015; Hofman, 2015). Sin embargo, el presupuesto disminuyó en 2016 en un 20\%. Es importante destacar que los recursos federales destinados para este programa continúan en disminución año a año y, ante la pandemia de la COVID-19, quedó estático como muchos otros. No obstante, en la economía de las familias, el Programa Pueblos Mágicos no ha detonado directamente, aunque representa un fuerte atractivo del turismo. En el caso de Izamal, el turismo cultural forma parte del atractivo de este. Enríquez et al. (2015, p. 83, citado en Sandoval \& Ortega, 2018, p.178) detallan que la comunidad (población) se encuentra como la penúltima beneficiaria del turismo, pero los servicios relacionados con este son los que obtienen mejores beneficios. Los pobladores observan que los beneficiarios directos son y serán los comerciantes, los que transitaron de las actividades econó- 
micas tradicionales, como el campo y las artesanías, al comercio, seguido del gobierno, en este caso del H. Ayuntamiento, y por último la ciudadanía. Cabe destacar que la ciudadanía interactúa con los turistas, resguardando su cultura y tradiciones. El turista lo que busca es conocer, saber cómo se comportan los pobladores y escuchar la forma de diálogo y expresión, siendo esta otra forma de participación de los pobladores.

De acuerdo con el INEGI (2019), en Izamal se tenía un registro de 224 unidades económicas (UE) dedicadas al servicio de preparación de alimentos a la carta o de comida corrida, integrando laboralmente a 648 empleados, con una media de 2,9 personas trabajando en cada una de ellas. Actualmente, se tienen 275 UE del mismo giro (INEGI, 2021), con un crecimiento del $18,6 \%$, teniendo en cuenta que en la pandemia de la COVID-19 esta actividad dirigida al turismo transitó hacia los pobladores y se acrecentó. Para el servicio de preparación de alimentos, se identificó que el turista opta por probar los platillos tradicionales de la localidad de Izamal como el salpicón, el deshebrado, el poc chuc, el mechado, entre otros, con el sabor particular de la elaboración a la leña (que son trozos de árboles secos, obtenidos a través del envejecimiento de los árboles). Lo anterior representa un ahorro en el consumo de gas en la preparación de alimentos.

En relación con el comercio de artesanías, que se dirige hacia el turista, se destaca que en 2019 se tenían 41 empresas y 42 en 2021, de acuerdo con el INEGI (2019/2021). La rotación de estos productos se ha efectuado en línea. Aunque esta actividad económica prevalece, lo hace de forma estática, con un bajo crecimiento en la localidad. De hecho, desde la perspectiva de la localidad, es la actividad económica que se ha abandonado paulatinamente. Por otra parte, el comercio de prendas de vestir, entre las que se cuenta la ropa típica, reporta registros de 29 y $34 \mathrm{UE}$, según los datos registrados por el INEGI (2019/2021). Esta actividad presentó un crecimiento del 15\%, su acceso para el turista se ha efectuado por medio de canales como internet y redes sociales. Es decir, el turismo sigue impactando positivamente en los ingresos económicos de los habitantes del municipio.

Arista et al. (2021) establecen que el Programa Pueblos Mágicos se conduce hacia un éxito a corto plazo, sin considerar los ámbitos sociales, económicos, de medioambiente y patrimonio, teniendo en cuenta en su gestión la postura desde lo local, es decir, a la población o ciudadanía inmersa en el territorio. Esto significa hacerla partícipe y dar a conocer el programa para identificarlo y valorarlo, con el fin de distinguir los beneficios que este trae en materia social y económica para los pobladores. ¿Por qué se genera esta falta de conocimiento de los beneficios del programa? Con base en la información que se obtuvo en el presente estudio, se considera que es debido a la ausencia de la cultura de divulgación de este, que permita orientar a la población hacia un mismo resultado (el 
incremento de la afluencia turística). El municipio de Izamal se incorporó al Programa Pueblos Mágicos en 2002, hace 19 años, es decir, el municipio no se excluye del programa en un corto plazo. Contrariamente a lo que señalan Arista et al. (2021), es un programa que ha permitido transitar la economía y los ingresos de los ciudadanos a partir de su presencia de forma positiva.

Sin embargo, Izamal no ha logrado consolidar los servicios y procesos turísticos en un escenario de crecimiento económico del municipio, lo que conduce a vincular la propuesta de Yee et al. (2019), que incita a dirigir los esfuerzos de los actores involucrados hacia nuevas alternativas, partiendo de un diagnóstico integral del municipio para un planteamiento concreto y correcto, en donde todos los actores involucrados se dirijan hacia un mismo rumbo, en especial la población (ciudadanía) y los empresarios.

\section{Conclusiones}

La percepción de los ciudadanos con respecto al Programa Pueblos Mágicos coincide, lo que ha incidido en el tránsito de las actividades tradicionales hacia el comercio y los servicios turísticos y ha influenciado de manera positiva la economía, incorporando de esa forma a la población económicamente activa (PEA) en estas actividades y mejorando los ingresos que se obtienen por el turismo. A pesar de que en la actualidad el flujo del turismo ha mermado, esto no ha logrado detener a las empresas, buscando que el giro comercial y de servicios continúe a través de otros espacios, siendo el comercio en línea y las redes sociales la fuente generadora principal de ingresos. Desde la perspectiva de los ciudadanos de Izamal, los beneficios recaen en los comerciantes o empresarios del municipio y en las autoridades, sin embargo, los ciudadanos participan en las actividades económicas que mencionan, ya que es en la parte comercial y de servicios enfocados al turismo en donde la población se ha incorporado con mayor fuerza, lo cual genera ingresos constantes en más del 30\% de los 13.775 ciudadanos que conforman la PEA-ocupada de Izamal (INEGI, 2020) y permite contribuir a los ingresos económicos familiares. La contribución y beneficio se observa de forma directa en la infraestructura de la población a partir de la percepción del sujeto de estudio, puesto que comparada con otras localidades que no poseen el distintivo de Pueblo Mágico, Izamal luce con colores homogéneos: las viviendas del primer cuadro de la ciudad tienen tonalidades amarillas. Además, la limpieza y la forma tradicional de vestimenta de los pobladores logra diferenciar al poblado de otros municipios de Yucatán. El $45 \%$ de los ciudadanos observan ingresos mejorados, siempre que sus actividades se asocien al comercio y a los servicios vinculados al turismo. También se identifica con la información recabada que la edad influye en la percepción de los resultados económicos desde el nombramiento como Pueblo Mágico hasta la fecha. Existe una inclinación que indica que a menor edad, mejor percepción del programa Pueblos Mágicos y de su 
influencia económica positiva hacia la localidad y hacia los ciudadanos.

\section{Propuestas o contribuciones}

A continuación, se presentan las siguientes propuestas:

Es importante la divulgación y el involucramiento de las instituciones, autoridades, empresarios y ciudadanos del municipio en el desarrollo de actividades culturales, sociales, ambientales y económicas derivadas de los beneficios económicos y de infraestructura obtenidos como parte del programa.

Hacer partícipes de las actividades a los habitantes del municipio no solamente invitándolos a participar, sino generando conciencia de las oportunidades que para ellos implica el poder desarrollar y ofrecer productos y servicios para la generación de ingresos alternos que contribuyan en la mejora de su calidad de vida y la de sus familias.

En época de pandemia (COVID-19), el flujo turístico fue nulo y la población está constatando la importancia de la afluencia turística de corte intermunicipal, nacional e internacional. Es entonces el momento para diseñar estrategias que incidan en el reposicionamiento de Izamal, para inclinarse hacia la mejoría de los productos y servicios que se ofrecen, así como para valorar las actividades tradicionales como la agricultura y la ganadería, que se han abandonado desde el nombramiento como Pueblo Mágico, y que hoy en día son las actividades que están permitiendo la permanencia económica de muchas familias de la localidad.

Es importante involucrar la participación de los pequeños productores familiares dentro de las actividades económicas asociadas al turismo, con la finalidad de mejorar los ingresos familiares y el consumo local de los productos.

Diseñar estrategias de marketing social que le permitan al municipio de Izamal seguir posicionándose a nivel nacional e internacional como Pueblo Mágico e incrementar la afluencia de turistas locales, nacionales y extranjeros.

Diseñar estrategias de capacitación para trabajar por cuenta propia, las cuales cubran el sector industrial y el sector de servicios existentes en el municipio de Izamal.

Inculcar en la población el valor del cuidado ambiental, que permita la limpieza e higiene de las viviendas, así como de las calles, depositando los residuos en los espacios correspondientes, ya que con la imagen de frescura e higiene se preservan los espacios y se incentiva la fluctuación de turistas hacia los sitios que posee el municipio, como el convento franciscano y las zonas arqueológicas que se encuentran próximas al centro de la ciudad.

Generar propuestas para la especialización de guías de turistas en el municipio, con el fin de que se cuente con la certificación de las instancias 
respectivas que validen la calidad de la información trasmitida al turista asiduo al municipio.

Crear operadoras locales certificadas que ofrezcan servicios turísticos y que brinden una oportunidad de trabajo a los habitantes del municipio.

Monitorización por parte de las autoridades de los recorridos en calesa, con la finalidad de salvaguardar y respetar la salud de los animales, además de garantizar el cuidado de los turistas durante el recorrido para preservar esta hermosa y antigua tradición.

Incentivar a la población para que sea partícipe en la construcción de propuestas que coadyuven con el crecimiento económico del municipio y con la integración moderada empresarial, ante la apertura de las empresas, respetando las indicaciones de los H. Ayuntamientos para la continuidad operativa y el cuidado de la salud de los empresarios, empleados y la población en general.

\section{Información complementaria}

\section{Agradecimientos}

Los autores agradecen a la Red Gestio y a la Red RedAyN, a través de la Dra. Luz del Carmen Morán Bravo, el establecimiento de la línea del turismo.

Contribuciones de autoría:

1. Karina Concepción González Herrera: genera la propuesta del resumen. Realiza el análisis docu- mental de la metodología, refleja y analiza los resultados y contribuye con las conclusiones y recomendaciones del presente material.

2. Aurea Licet Castillo Gallegos: realiza el contexto teórico, retroalimenta la metodología, contribuye con la conclusión y aportación en las propuestas y contribuciones.

3. Karla Gabriela Gómez Galaz: genera la propuesta del resumen. Realiza el análisis documental de la metodología y del marco teórico, refleja y analiza los resultados y contribuye con las conclusiones y recomendaciones del presente material.

4. José Jesús Hernán Negrón Noh: genera la propuesta del resumen. Realiza el análisis documental del marco teórico, refleja y analiza los resultados y contribuye con las conclusiones y recomendaciones del presente material.

\section{Conflictos de interés}

Los autores del presente artículo establecen que no presentan conflicto de intereses en relación con la revista y sus editores.

\section{Referencias}

Arista-Castillo, L., Hiriart-Pardo, C. A., \& Barrera-Fernández, D. (2020). Resiliencia y conservación en Pueblos Mágicos de México. Los casos de Pátzcuaro y Mexcaltitán. Bitácora Urbano Territorial, 31(1), 195-210. https://doi. org/10.15446/bitacora.v31n1.86340 
Alcocer-Kuk, M. J., Ulibarri-Benítez, H. A. \& Pereyra-Chan, A. M. (2019). El contexto de Izamal y Valladolid en su denominación como Pueblos Mágicos. Revista del Centro de Graduados e Investigación. Instituto Tecnológico de Mérida, 34(79), 09-17. https://bit.ly/3jWFZL8

Barbero, M. I. (Coord.), Vila E. \& Suárez J. C. (Ed. Enero 2006, $1^{\text {a }}$ Reimpresión): Psicometría. Unidades Didácticas. Madrid: UNED.

Cabrera, M. F. (2017). Diagnóstico sobre el nivel de generación de negocios inclusivos en la localidad de Kennedy en Bogotá y su impacto en el desarrollo local. Revista GEON (Gestión, Organizaciones Y Negocios), 4(1), 116-134. https://doi.org/10.22579/23463910.49

Consejo de Nacional de Población [CONAPO]. (2015). Índice de marginación por entidad federativa 1990-2015. https://bit.ly/3yXnbio

Consejo de Nacional de Población [CONAPO]. (2015). Índice de marginación por municipio 1990-2015. https://bit. ly/3neSTWF

Enríquez, J., Guillén, M., Valenzuela, B., \& Jaime M. E. (2015). Magdalena de Kino, Sonora. Percepciones de la población con respecto al patrimonio cultural y el turismo. Topofilia. Revista de Arquitectura, Urbanismo y Territorios, V(1), 67-88. https://bit.ly/3yw9v83

Fernández, P. M. (2016). Una revisión del Programa Pueblos Mágicos. CULTUR: Revista de Cultura e Turismo. 10(1), 3-34. https://bit.ly/3A15PTG

Flores-Pacheco, N. A. (2019). Desarrollo regional: motivo de participación en proyectos de turismo gastronómico. El caso de San Pablo Villa de Mitla, Oaxaca. Estudios sociales. Revista de alimentación contemporánea y desarrollo regional, 29(53), e19685. https://doi. org/10.24836/es.v29i53.685

Güidi, G. (2016). Desarrollo local y turismo: ejes de articulación entre conceptos. Red Sociales, Revista del Departamento de Ciencias Sociales, 03(06), 06-21. https://bit.ly/3jThPRq

Hofmann, A. (2015). Pueblos mágicos de la magia al desarrollo local. Buen Gobierno, (19),106-120. https://bit.ly/3BYrZ9P

Hernández, S. R., Fernández, C. C., \& Baptista, L. P. (2014). Metodología de la Investigación (6. ${ }^{a}$ edición). McGrawHill.

Instituto Nacional de Estadística y Geografía [INEGI]. (2020). Banco de indicadores. Izamal, Izamal, 31040. https:// bit.ly/3hew90A

Instituto Nacional de Estadística y Geografía [INEGI]. (2019). Censos Económicos 2019. Datos, Tabulados. https://bit. ly/3E1qSrK

Instituto Nacional de Estadística y Geografía [INEGI]. (2020). Censos de Población y Vivienda 2020. Microdatos. https://bit.ly/3zVilyD

Instituto Nacional de Estadística y Geografía [INEGI]. (2020). Número de habitantes por municipio. Población. https:// bit.ly/3hhkROm

Instituto Nacional de Estadística y Geografía [INEGI]. (2021). Directorio Estadístico Nacional de Unidades Económicas [DENUE]. https://bit.ly/3DZdHYo

Lacave, R. C., Molina, D. A. I., Fernández, G. M., \& Redondo, D. M. Á. (enero de 2016). Análisis de la fiabilidad y validez de un cuestionario docente. ReVisión, 9(1), 23-36. https://bit.ly/38R3tLd

Pérez-Ramírez, C. A., \& Antolín-Espinosa, D. I. (2016). Programa pueblos mágicos y desarrollo local: actores, dimensiones 
y perspectivas en El Oro, México. Estudios Sociales. Revista de alimentación contemporánea y desarrollo regional, 25(47), 218-242. https://bit.ly/3tu2ZnU

Ramos, J. L. (2015). Pierden Magia por pobreza y violencia. Pueblos Mágicos [presentación de dispositivas] Issue. https://bit.ly/3A8hPCl

Sandoval, N. L. \& Ortega, R. M. E. (2018). Índice de capital social para el Pueblo Mágico de Tapijulapa, Tabasco. Revista de El Colegio de San Luis, VIII(15),171201. https://bit.ly/3hetic]

SECTUR, SEFOTUR, UADY. (S. f.). Municipio de Izamal, Yucatán. Diagnóstico de competitividad y sustentabilidad de los pueblos mágicos. Website. Recuperado de: https://bit.ly/3z1GVCg

Secretaría de turismo [SECTUR]. (03 de enero de 2014). Pueblos mágicos. Gobierno de México. https://bit. ly/3E3eQya

Secretaría de turismo [SECTUR]. (2014). Guía de incorporación y permanencia, Pueblos mágicos. Gobierno de México. https://bit.ly/2YEXzzL

Secretaría de turismo [SECTUR], Secretaría de Fomento Turístico [SEFOTUR]
\& Universidad Autónoma de Yucatán [UADY]. (03 de enero de 2014). Diagnóstico de competitividad y sustentabilidad de los pueblos mágicos. Municipio de Izamal, Yucatán. Sefotur. https://bit. ly/2X12DsL

Segovia-Hidalgo, S., Peña-Estrada, C.C. \& Velázquez-García, L. A. (2019). Pueblos Mágicos: Economic And Local Development Analysis On The Touristic Impact. Journal of Tourism and Heritage Research, 2(4), 288-307. https://bit. ly/2VwqZu0

Tourón, J. (1989). La validación de constructo: su aplicación al CEED (cuestionario para la evaluación de la eficacia docente). Bordón, 41(4), 735-756. https://bit.ly/3hfmrz]

Verdugo, M. V. \& Cal, M. I. (2010). Valoración de la enseñanza: SEEQ. Revista de Formación e Innovación Educativa Universitaria, 3(4), 182-193. https://bit. ly/3to62hk

Yee, S. Z., Borges, R. \& Ángeles, M. (2019). Parámetros para la transición a un nuevo modelo de Pueblos Mágicos. El caso de Loreto, Baja California Sur. El Periplo Sustentable, (37), 334-355. https://doi.org/10.36677/elperiplo. v0i37.10410 